\title{
On Possible Models of Attention in Signal Detection
}

\author{
RoNAII) IIÜBNI:R
}

Uninersity of California ar Berkeley.

\begin{abstract}
This paper is concerned with two different mechanisms of altention in auditory signal detection. One alters the width of a Gaussian filter (or attention band), whereas the other changes the tuning. that is. the placement of its center. To formatize these mechanisms, we first consider auditory energy-detection. To explore the eflect of a randomly tuned filter, both uniform and normal density distributions of its center frequency are investigated. Formulas for predicting signal detection performannce in $2 \mathrm{FC}$ experiments are derived for the different cases resulting from thesc hypotheses, and cxamples of psychometric functions arc compulcd. The results reveal striking differences between the two mechanisms. Widening the auditory filter not only reduces sensitivity but also stecpens the resulting psychometric functions. In contrast, changing the filter tuning caluses psychometric functions to flatten.
\end{abstract}

\section{INTROI)UCTION}

Decreasing performance in auditory signal detection situations with frequency uncertainty led investigators to question how subjects distribute their attention across the frequency range if they are uninformed about the frequency of the signal. Several generalizations of ideal observer models, mainly the energy-detection, emerged Io predicl lhe oulcome in llese complex lasks (sec Swels, 1984, for a review). The medels vary in the number of lithers (or hands) they propose and the way liller outpuls are eombined. Usually, such models are divided in two classes: single-band and multiple-band models.

The consequences of both classes of models have been derived in order to distinguish between them empirically. So far, however, experiments have failed to establish which class of models is more appropriate. Accordingly, this paper is concerned with deriving additional features which can help to discriminate the two classes of models. To attain this objective the underlying ideas are specified in greater mathematical detail. This approach has been emphasized by Shiffrin (1988), who pointed out that detailed mathematical models of the attention processes are needed to provide firm answers. To motivate the derivations we first describe the relevant aspects of auditory signal detection and their respective experimental paradigms.

Reprint requests should be addressed to Ronald Hübner. Institut für Psychologic, Techniseiti Universitï Braunschweig. Gaussirassc 23, D-3300 Braunschweig, Germany. 
To begin, in a simple auditory signal detection experiment, a listener is typically asked to detect a known sinusoidal signal in background noise. It turns out that detection performance in this relatively simple situation depends mainly on the signal-to-noise ratio. However, if the listener is also unfamiliar with the frequency of the signal, additional detection difficulties arise even when signals are several decibels above the simple threshold. It has been argued that listeners uncertain about the signal frequency do not know what frequency to listen to. Typical measures taken to eliminate this uncertainty and its associated loss of sensitivity, involve either switching off the noise at the beginning of the experimental session, or presenting the first few signals at a relatively high level such that they are clearly audible to the listeners. (For an experimental investigation of the latter strategy, see Gundy, 1961). This demonstrates that providing information about the signal frequency is helpful in directing the listener's attention to the respective frequency region and improving performance.

Attention effects have also been investigated experimentally by deliberately exposing listeners to frequency uncertainty throughout the experiment. It appears that detection performance consistently decreases as frequency uncertainty increases. Several models of selective attention have been proposed to account for such decrements in performance (see Swets, 1984). As mentioned earlier, the concept of auditory filtering is central to these models.

Filtering is associated with one of the most striking features of the human auditory system, its frequency resolution capability. Ohm (1843) was among the first who systematically observed this feature. Later, von Helmholtz (1863) suggested a bank of band-pass filters as a model to account for frequency resolution. Subsequently, Fletcher (1940) provided psychophysical data strongly supporting auditory filters and proposed a method for their quantification. While investigating masking effects of noise varying in bandwidth, he discovered that only a limited band of noise around the signal frequency is effective in masking the signal. Moreover, his data indicated that the masking band increases with signal frequency. These results suggest that listeners listen through a band-pass filter or a "critical band," as Fletcher (1940) called it, centered on the signal frequency.

Since then, attempts have been made to determine the "true" auditory filter width. However, different methods lead to different estimates of the bandwidth. Moreover, Green (1960), using noise as both signal and masker, concluded that the filter width is variable. Although some estimates of critical bands may indicate their minimum bandwidth, they seem to be adjustable beyond this minimum. Therefore, it has been suggested that the critical bandwidth may not be fixed, but can be adjusted "under intelligent control" (Green, 1960; Swets, Green, \& Tanner, 1962).

Although the idea of a variable critical band provides a possible mechanism of auditory attention, earlier single-band and multiple-band models of attention assumed fixed bandwidths. The first single-band model was introduced by Tanner, Swets, and Green (1956) (cited in Swets, 1963). They assumed that listeners employ a single band-pass filter and are capable of controlling its location. Listeners can voluntarily tune the filter to certain frequencies. If the filter is moved from one 
frequency location to another, it is assumed that it also sweeps through the intermediate frequencies.

If the listener is uncertain about the signal frequency, he or she probably does not tune the filter optimally. Attention is not focused at the correct location. Consequently, the listener's sensitivity is reduced. To test this and other predictions an experimental situation is frequently used in which the signal may have one of two equally likely frequencies in a given trial. Assume a two-interval forced-choice (2IFC) procedure, that is, each trial consisting of two time intervals only one of which contains the signal. The listener's task is to indicate the interval he or she believes to have contained the signal. Tanner et al. (1956) who conducted such an experiment, varied the frequency separation of the two possible signals. Results were in line with a single-band model. Detection performance decreased as a function of the frequency separation, but approached a minimum when the separation was sufficiently large. This minimum has been interpreted as suggesting that listeners are incapable of sweeping the filter from one location to the other during a brief signal interval. In this case the listeners can attend to only one frequency during each trial. If they select this frequency randomly, the probability $P_{2}\{C\}$ of being correct across trials is

$$
P_{2}\{C\}=0.5 P_{1}\{C\}+0.5(0.5),
$$

where $P_{1}\{C\}$ is the probability of being correct in a one-frequency situation.

One of the earliest multiple-band models was proposed by Green (1958). He assumed that listeners can utilize more than one band-pass filter simultaneously. The output of different bands is then combined via some form of linear combination. Thus, if the listener is asked to detect a signal with one of two possible frequencies, he or she listens through two filters; as a result, the effective masking noise is increased as compared to the fixed frequency situation. Hence, the signalto-noise ratio is decreased and, consequently, also detection performance.

Several experiments have been conducted testing single-band and multiple-band models as alternatives (e.g., Creelman, 1960; Swets, Shipley, McKey, \& Green, 1959; Swets \& Sewall, 1961; Veniar, 1958). Unfortunately, the data are inconclusive. Some experiments support the single-band models, others multiple-band models, and a few support even both. In addition, qualitative individual differences have been reported. Some listeners behaved as though they were listening through a single band, while others seemed to listen through multiple bands. There are other results posing difficulties for both classes of models. For instance, Green (1961) conducted an experiment in which the signal could have any frequency ranging from 500 to $4000 \mathrm{~Hz}$. The decrement in detection performance was far less than predicted by either model. To explain this result, Green suggested that there is some initial uncertainty even in detecting a single frequency. Presentation of a stimulus with a fixed frequency does not guarantee that the listener knows the frequency 
exactly. In an experiment by Shipley (1959) the signal could have relatively high or low levels chosen at random. The results showed that detection performance improved after a correct response to a high-level signal in comparison with the performance following a correct response to a low-level signal. This indicates that a preceding high-level signal may serve as a cue, helping to define the signal frequency.

Interestingly, frequency uncertainty introduced by using unspecified signals can be, at least partly, compensated by presenting a cue to the listener indicating which frequency comes next. This has been demonstrated by Swets and Sewall (1961). In one situation they used a sinusoidal cue with the same frequency as the signal. In another, different lights were presented as cues for certain frequencies. Although cues failed to remove uncertainty entirely, improvement was appreciable. Furthermore, Johnson and Hafter (1980) showed that cues can improve performance even when the listener has some knowledge of signal frequency.

Considering all these results, the question arises as to whether there may be additional features differentiating the two model classes not yet taken into account. It will be shown here that there are indeed striking differences not noted so far. We proceed by assuming that traditional single-band models are not quite realistic. Then we suppose assuptions we deem more suitable and look at the outcomes.

If the notion of a single moving filter is adopted, it is unlikely that the filter will be placed optimally after moving from one frequency location to another. It is more reasonable to suppose that signal frequencies are generally not known exactly. As a consequence, it is very unlikely that the center frequency of the filter will be located precisely on the signal frequency in every trial. Admittedly, a slight misplacement of the filter should not produce a decrement in detection if the filter has a rectangular shape. However, if the filter has sloping skirts then there will be attenuation close to center frequency. Even a small misalignment will cause the signal-to-noise ratio to decrease.

The assumption of a rectangular filter may be inappropriate. Obtained data suggest the shape of the auditory filter to be approximately Gaussian (Patterson, 1974) or exponential (Patterson, Nimmo-Smith, Weber, \& Milroy, 1982). Accepting this result, the consequences for the behavior of the single-band model can be derived. This is done by investigating the effect of misplacing the center frequency of such a filter. As mentioned before, to answer this question in detail it is necessary to quantify several underlying concepts. Thus, additional assumptions must be imposed. First, we need to know or to assume how the listener processes each stimulus. In what follows we assume an energy-model of auditory signal detection (cf. Green \& Swets, 1966). Additionally, two different probability distributions of the filter's center-frequency placement are considered: uniform and normal. Our results are then contrasted with alternative models of attention: varying the filter width or the number of filters. We begin by describing the energymodel of auditory signal detection. 


\section{Energy-Model of Auditory Signal Detection}

For a 2IFC situation detection theory assumes that each of the two intervals contains a sample of one of the two random variables $U_{s}$ and $U_{n}$. Here, the random variable $U_{s}$

alone. The observer chooses the interval containing the larger sample. Formally, let $P\{C\}$ denote the probability of a correct response. Then it is assumed that

$$
P\{C\}=P\left\{U_{s}>U_{n}\right\}=\int_{-\infty}^{\infty} F_{n}(u) f_{s}(u) d u,
$$

where $F_{n}(u)=\int_{-\infty}^{u} f_{n}(y) d y$ is the distribution function of $U_{n}$ and $f_{s}(u)$ is the probability function of $U_{s}$.

Obviously, to predict the behavior of an observer it is necessary to specify the probability distributions of noise and signal plus noise. Hence, we must know how the observer processes the signal. One assumption is that the energy content of each sample is extracted and the two samples are compared. To do the comparison each sample is passed through an identical filter and the energy output is noted. This energy-model has served as a basis for most single-band and multiple-band treatments of attention. To compute energy distributions of noise and signal plus noise we need to specify a formal representation of the respective waveforms.

Consider a waveform $x(t)$ which can be either band-limited noise $n(t)$ or a signal plus such noise $s(t)$. Further, let the time interval on which the waveform is defined be represented by the closed real interval $[0, T]$. Then, a representation of the waveform is given by

$$
\hat{x}(t)=\sum_{k=1}^{\infty} a_{k} \cos \frac{2 \pi}{T} k t+b_{k} \sin \frac{2 \pi}{T} k t, \quad 0 \leqslant t \leqslant T, k \text { integer, }
$$

where

$$
a_{k}=\frac{2}{T} \int_{0}^{T} x(t) \cos \frac{2 \pi}{T} k t d t
$$

and

$$
b_{k}=\frac{2}{T} \int_{0}^{T} x(t) \sin \frac{2 \pi}{T} k t d t
$$

(cf. Green \& Swets, 1966). This representation is a Fourier series and provides an approximation $\hat{x}(t)$ of the continuous waveform $x(t)$. The lowest frequency as well as the frequency spacing is $1 / T$. For the band of noise $n(t)$ it is assumed that the coefficients $a_{k}$ and $b_{k}$ are independent Gaussian variables with $\mu=0$ and $\sigma_{k}^{2}=N_{0} / T$, where $N_{0}$ denotes the noise power in a $1-\mathrm{Hz}$ band (for more details see Green \& Swets, 1966). In the present paper the noise band is assumed to cover the hearing range. Outside this band the coefficients are zero. 
After Parseval's theorem the power in the waveform approximation $\hat{x}(t)$ is

$$
\frac{1}{T} \int_{0} \hat{x}^{2}(t) d t=-\frac{\sum_{k=1}}{}\left(a_{k}^{2}+b_{k}^{2}\right) .
$$

If the waveform $x(t)$ is noise alone, the energy of the approximation is a random variable and is given by

$$
U_{n}^{*}=\int_{0}^{T} \hat{n}^{2}(t) d t=\frac{T}{2} \sum_{k=1}^{\infty}\left(a_{k}^{2}+b_{k}^{2}\right) .
$$

Standardizing the constants and letting $\alpha_{k}=a_{k} / \sqrt{N_{0} / T}, \beta_{k}=b_{k} / \sqrt{N_{0} / T}$ one obtains

$$
\frac{2 U_{n}^{*}}{N_{0}}=\sum_{k=1}^{\infty}\left(\alpha_{k}^{2}+\beta_{k}^{2}\right)
$$

After passing the noise through some arbitrary filter with a squared-magnitude function $|H(k / T)|^{2}$ one obtains (see Patterson \& Henning, 1977)

$$
\frac{2 U_{n}}{N_{0}}=\sum_{k=1}^{\infty}\left(\alpha_{k}^{2}+\beta_{k}^{2}\right)|H(k / T)|^{2} .
$$

If a sinusoidal signal with amplitude $A$ and in sine phase is added to the noise, it is necessary that its frequency $f_{0}$ be an integral multiple of $1 / T$.

a positive integer such that $f_{0}=k_{0} T$. The energy of the approximation for this case is given by

$$
U_{s}^{*}=\int_{0}^{T} \hat{s}^{2}(t) d t=\frac{T}{2}\left[\sum_{k=1}^{\infty}\left(a_{k}^{2}+b_{k}^{2}\right)+A^{2}+2 A\left(a_{k_{0}} \sin \theta+b_{k_{0}} \cos \theta\right)\right] .
$$

Standardizing and filtering leads to

$$
\begin{aligned}
\frac{2 U_{s}}{N_{0}}= & \sum_{k=1}^{\infty}\left(\alpha_{k}^{2}+\beta_{k}^{2}\right)|H(k / T)|^{2} \\
& +\left[\frac{T A^{2}}{N_{0}}+2 A \sqrt{T / N_{0}}\left(\alpha_{k_{0}} \sin \theta+\beta_{k_{0}} \cos \theta\right)\right]\left|H\left(f_{0}\right)\right|^{2} .
\end{aligned}
$$

Note that the cross-product terms will have an expectation of zero over the trials. Because the $\alpha_{k}$ and $\beta_{k}$ are independent standardized normal variables, their squares are distributed as $\chi^{2}$. Consequently, also $\left(\alpha^{2}+\beta^{2}\right)$ is distributed as $\chi^{2}$ with two degrees of freedom. Thus, the distribution of noise-alone (Eq. (4)) has mean

$$
\mathscr{E}\left(\frac{2 U_{n}}{N_{0}}\right)=2 \sum_{k=}^{\infty}|H(k / T)|^{2}
$$


and variance

$$
\operatorname{Var}\left(\frac{2 U_{n}}{N_{0}}\right)=4 \sum_{k=1}^{\infty}|H(k / T)|^{4} .
$$

Signal-plus-noise produces a distribution with mean

$$
\mathscr{E}\left(\frac{2 U_{s}}{N_{0}}\right)=2 \sum_{k=1}^{\infty}|H(k / T)|^{2}+\frac{T A^{2}}{N_{0}}\left|H\left(f_{0}\right)\right|^{2}
$$

and variance

$$
\operatorname{Var}\left(\frac{2 U_{s}}{N_{0}}\right)=4 \sum_{k=1}^{\infty}|H(k / T)|^{4}+\frac{4 T A^{2}}{N_{0}}\left|H\left(f_{0}\right)\right|^{4} .
$$

Fortunately, when the number of components entering into the sums (4) and (5) is not too small, we can assume that the distributions are approximately normal (Lindeberg's theorem, Feller, 1966). This makes the calculation of the proportion of correct responses in a 2IFC task quite easy. Because it is assumed that observers select the interval containing the larger energy sample, they respond correctly if and only if the larger sample is drawn from the signal-plus-noise distribution. However, the probability that $U_{s}>U_{n}$ is the same as the probability that $U_{s}-U_{n}>0$. Because the difference of normal variables is also normally distributed, the percentage correct is given by

$$
P\{C\}=\Phi(z)=\frac{1}{\sqrt{2 \pi}} \int_{-\infty}^{z} e^{-x^{2} / 2} d x
$$

where

$$
\begin{aligned}
z & =\frac{\mathscr{E}\left(2 U_{s} / N_{0}\right)-\mathscr{E}\left(2 U_{n} / N_{0}\right)}{\sqrt{\operatorname{Var}\left(2 U_{s} / N_{0}\right)+\operatorname{Var}\left(2 U_{n} / N_{0}\right)}}, \\
& =\frac{\left(T A^{2} / N_{0}\right)\left|H\left(f_{0}\right)\right|^{2}}{\sqrt{8 \sum_{k=1}^{\infty}|H(k / T)|^{4}+\left(4 T A^{2} / N_{0}\right)\left|H\left(f_{0}\right)\right|^{4}}} .
\end{aligned}
$$

Using the fact that the energy $E_{s}$ of a sinusoidal signal is $T A^{2} / 2$ one obtains from Eq. (8):

$$
z=\frac{\left(E_{s} / N_{0}\right)\left|H\left(f_{0}\right)\right|^{2}}{\sqrt{2 \sum_{k=1}^{\infty}|H(k / T)|^{4}+\left(2 E_{s} / N_{0}\right)\left|H\left(f_{0}\right)\right|^{4}}} .
$$

For reasons that will become clear later we convert Eq. (9) to a function of the filter gain at the signal frequency $y=H\left(f_{0}\right)$ :

$$
z(y)=\frac{\left(E_{s} / N_{0}\right)|y|^{2}}{\sqrt{2 \sum_{k=1}^{\infty}|H(k / T)|^{4}+\left(2 E_{s} / N_{0}\right)|y|^{4}}} .
$$


Let us consider the special case of a rectangular filter of width $W$ and centered at the signal frequency, that is,

$$
H(f)= \begin{cases}1, & \text { for } f_{0}-W / \\ 0, & \text { elsewhere }\end{cases}
$$

Since $H\left(f_{0}\right)=1$, Eq. (9) simplifies to

$$
z=\frac{\left(E_{s} / N_{0}\right)}{\sqrt{2 W T+2 E_{s} / N_{0}}} .
$$

Note that the energy of the signal as well as the spectral density of the noise are determined by the experimenter. The variables set by the auditory system are the auditory filter width and shape and the auditory integration time $T$.

It should be noted that there is at least one alternative method for deriving the energy distributions. This approach has been articulated by McGill (1967a, 1967b). He started with known energy distributions of narrow-band noise and signal plus noise. Then he constructed wide-band noise by adding up a specific number of narrow-band noise sources spaced orthogonally in frequency, producing the respective energy distributions.

\section{EfFect of Random Filter Position}

In this section we consider the effects of introducing additional assumptions to single-band models. More precisely, we investigate what happens when the filter is positioned randomly around the signal frequency rather than exactly on it. As mentioned in the Introduction, it is unlikely that filter placement is exact if one assumes a moving filter.

Throughout the following derivations, we assume that filter position varies randomly from trial to trial, but remains fixed within a trial. Therefore, the position of the center frequency of the filter can be represented by a random variable $X$. To calculate the effect of a randomly positioned filter the distribution of positions must be specified. Here, it is assume that $X$ has either a uniform or a normal distribution and that their respective means are identical to the signal frequency.

Random filter position implies that filter gain at the signal frequency is also a random variable, labeled here $Y$. Consequently, effective signal amplitude and energy become random variables as well. The effective signal amplitude depends on the gain of the filter at the signal frequency which is a function of filter shape.

Because this paper deals exclusively with symmetric filter shapes and symmetric density functions it is convenient to define a standardized frequency $x$ by

$$
x=\frac{\left|\int-\int_{0}\right|}{f_{0}},
$$


where $f_{0}$ denotes the signal frequency and $f$ denotes the unstandardized frequency in $\mathrm{Hz}$. Standardization shifts the origin to the signal frequency and maps the whole frequency range into positive reals. Accordingly, the standardized filter width will be denoted by $W_{s}$.

A great deal of effort has been directed at measuring auditory filter shape (for a review see Patterson \& Moore, 1986). A class of filter shapes that seems reasonable has been suggested recently by Patterson et al. (1982). It involves a slightly modified exponential shape called rounded-exponential (roex). The simplest filter form of this class is a one-parameter roex,

$$
H(x)=(1+b x) e^{-b x},
$$

where $b$ is a parameter determining the filter width. In this paper, however, an equivalent Gaussian filter is adopted for mathemetical convenience:

$$
H(x)=e^{-x^{2} / v} .
$$

Its equivalent rectangular bandwidth in standardized frequency is $\sqrt{v \pi}$. Figure 1 illustrates the filter shapes of a roex and a Gaussian filter each with an equivalent rectangular bandwidth of 0.4 . As can be seen, the filter shapes are quite similar. At least, they are sufficiently close for our purposes. In fact, a Gaussian filter of this type was originally proposed by Patterson (1974).

The gain of the Gaussian filter is continuous. Consequently, the corresponding

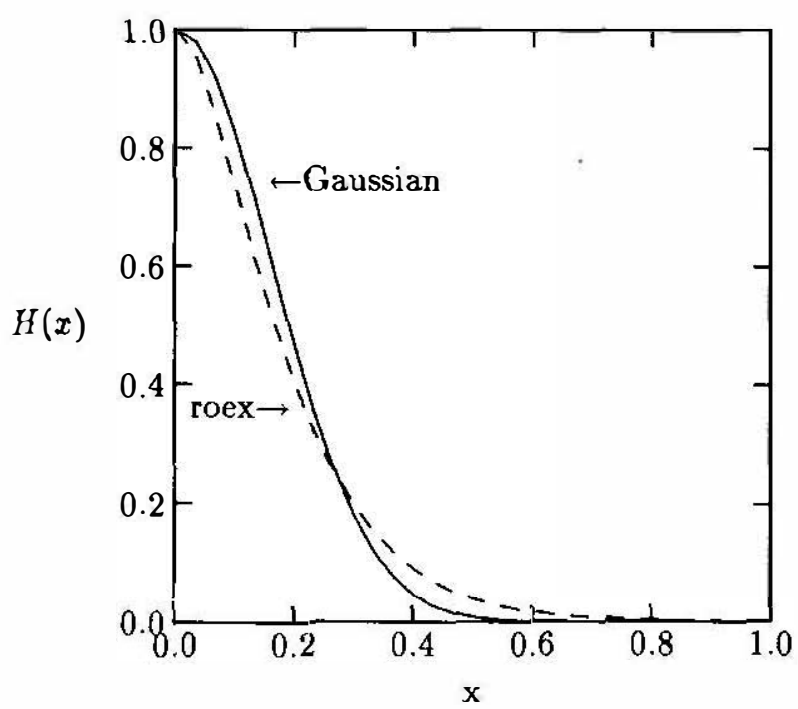

Fig. 1. Filter shapes of a Gaussian and a roex lilter with identical equivalent rectangular bandwidths. 
random variable $Y$ is also continuous. The proportion of correct responses in a 2IFC task is given by

$$
P\{C\}=\int_{-\infty}^{+\infty} P\{C \mid Y=y\} g(y) d y .
$$

Our task is to derive the probability density function $g(y)$ based on the distribution of the center frequency of the filter. In the next subsection a uniform distribution is considered, and in the subsequent subsection a normal distribution.

\section{Uniform POSiTION Distribution}

In this subsection it is assumed that the position of the filter center frequency is uniformly distributed in the standardized interval $(0, m)$. That is, the corresponding random variable $X$ has the density function:

$$
f(x)= \begin{cases}\frac{1}{m}, & \text { if } \quad 0<x<m, \\ 0, & \text { elsewhere. }\end{cases}
$$

To derive the density distribution of the random variable $Y$ note that the filter is a strictly decreasing function of $X$. The function $H$ maps the interval $(0, m)$ onto the interval $(H(m), 1)$. Therefore, the density of $Y$ is given by (cf., for example, Hogg \& Craig, 1970)

$$
g(y)= \begin{cases}f\left[H^{-1}(y)\right]\left|\frac{d H^{-1}(y)}{d y}\right|, & \text { if } H(m)<y<1, \\ 0, & \text { elsewhere }\end{cases}
$$

where $H^{-1}$ denotes the inverse function of $H$ and $|x|$ is the absolute value of $x$.

The inverse function of the Gaussian filter is

$$
H^{-1}(y)=\sqrt{v[-\ln y]}, \quad 0<y<1 .
$$

Consequently, the density function of $Y$ is

$$
g(y)=\frac{\sqrt{v}}{2 m y \sqrt{[-\ln y]}}, \quad H(m)<y<1 .
$$

Substituting this expression in Eq. (12) and applying Eq. (10) finally gives

$$
P\{C\}=\int_{H(m)}^{1} \Phi[z(y)] \frac{\sqrt{v}}{2 m y \sqrt{-\ln y}} d y .
$$




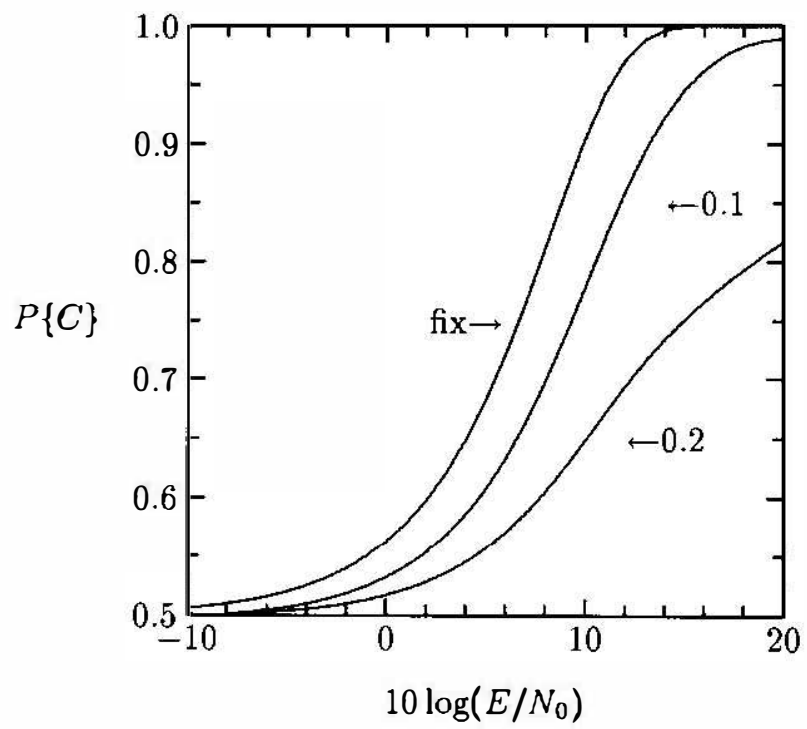

FIG. 2. Psychometric functions of an energy detector with a uniformly varying Gaussian filter. The curve labeled "fix" corresponds to a non-varying filter. The remaining curves correspond to variation ranges of $m=0.1$, and $m=0.2$.

This formula leads to the psychometric functions depicted in Fig. 2, corresponding to variation ranges $m$ of 0.1 and 0.2 . Throughout this paper a time constant of $T=0.2$ is used. The function labeled "fix" corresponds to a fixed filter and serves for comparison. Performance of a randomly moving filter can never be superior to the performance of a fixed filter centered on the signal frequency. As can be seen, random placement of the Gaussian filter decreases slope.

\section{Normal Position Distribution}

Although one can think of situations in which a uniform distribution may be appropriate, a normal position distribution is usually more suitable. One might suspect that cueing leads to normally distributed filter placements. Normality implies that center frequency can be moved to any location in the normalized interval $(0, \infty)$. The important parameter is the variance of the position distribution.

If the center frequency of the filter is normally distributed, the function $H$ maps the interval $(0, \infty)$ onto the interval $(0,1)$. Since only positive reals are considered, the density distribution of $X$ is

$$
f(x)=\frac{2}{\sqrt{2 \pi} \sigma} e^{-x^{2} / 2 \sigma^{2}}, \quad 0<x<\infty
$$


The reasoning applied in the previous section can now be used to derive the density function of $Y$. We have

$$
f\left[H^{-1}(y)\right]=\frac{2}{\sqrt{2 \pi} \sigma} e^{v \ln . / 2 \sigma^{2}}, \quad 0<y<1,
$$

which leads to the density function of $Y$,

$$
g(y)=\frac{\sqrt{v}}{y \sigma \sqrt{-2 \pi \ln y}} e^{v \ln y / 2 \sigma^{2}}, \quad 0<y<1 .
$$

Finally, one obtains

$$
P\{C\}=\int_{0}^{1} \Phi[z(y)] \frac{\sqrt{v}}{y \sigma \sqrt{-2 \pi \ln y}} e^{v \ln y / 2 \sigma^{2}} d y .
$$

Examples of psychometric functions reflecting these assumptions are shown in Fig. 3. The function corresponding to the fixed filter is identical to that in Fig. 2. Functions corresponding to standard deviations of 0.05 and 0.1 display the behavior already encountered in the case of a uniform distribution: slope decreases as variance increases.

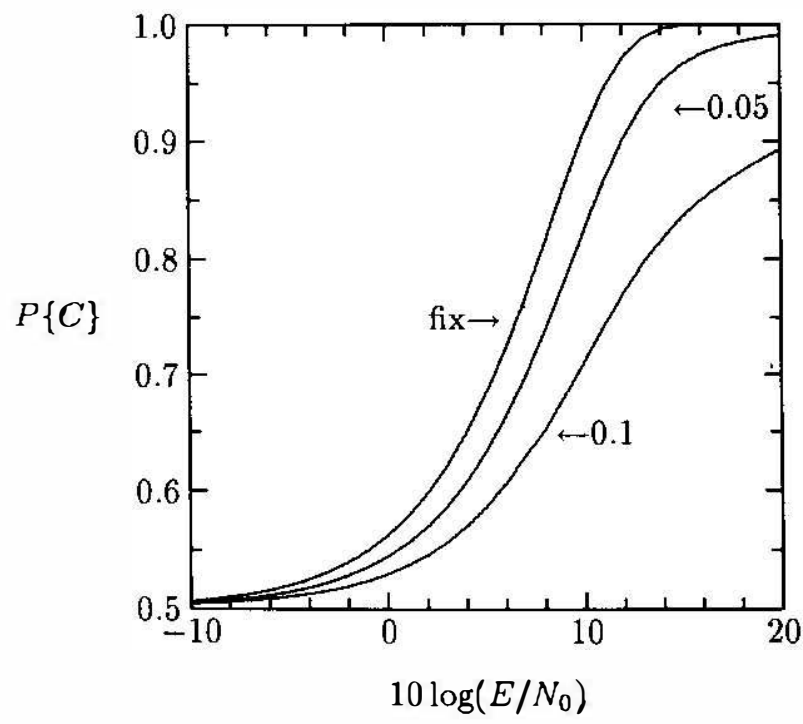

Fig. 3. Psychometric functions of an energy detector with a normally varying Gaussian filter. The curve labeled "fix" corresponds to a non-varying filter. The remaining curves correspond to standard deviations of the position distribution of 0.05 , and 9.1 . 


\section{The Effect of Increasing the Width or Number of Filters}

Arguments thus far have been concerned with various assumptions related to the single-band model and with the consequences if mistuning occurs with a single moving filter. This section is concerned with alternative models. As mentioned in the Introduction, broadening a single filter or utilizing multiple filters can be used equivalently to predict decreases in performance under uncertainty.

The first question investigated in this section is how the performance of an energy detector is affected by increasing its filter width. Suppose $2 W T>10$, so that the percentage correct in a 2IFC experiment can be approximated via formula (6). For convenience, only rectangular filters are considered in this section. No fundamental differences are obtained with a Gaussian filter. Varying the width $W$ of the rectangular filter produces different psychometric functions as illustrated in Fig. 4. The functions depicted correspond to filter widths of 20,50 , and $600 \mathrm{~Hz}$. As one would expect, widening of the filter shifts the psychometric function to the right; that is, the detector loses sensitivity. This is similar to the effect of mistuning a single filter. The interesting difference, however, is that the slope of the psychometric function increases with filter bandwidth. This result is contrary to the decreasing slopes obtained by mistuning a single filter.

It is worth pointing out that the same result is obtained with the alternative noise and signal-plus-noise distributions preferred by McGill (see Green \& McGill, 1970).

Next, the effect of utilizing more than one filter is investigated. Multiple-band models argue that the auditory system uses more than one filter in complex signal

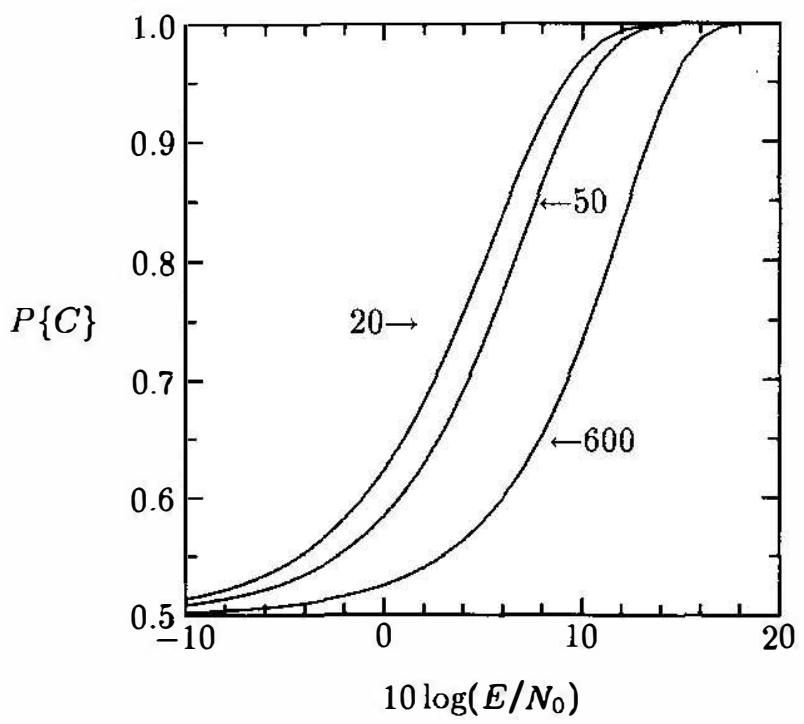

Fig. 4. Psychometric functions of an energy detector with a non-varying rectangular filter. The curves correspond to filter widths of 20,50 , and $600 \mathrm{~Hz}$. 
detection situations. Some multiple-band models were inspired by Green's (1958) statistical summation model. The latter was originally worked out to predict detection of multi-component signals. It was applied subsequently to detection under conditions of uncertainty (see Creelman, 1960). Consider, for example, a two-filter model with two random variables $X\left(f_{1}\right)$ and $X\left(f_{2}\right)$, each describing the output of a single filter. Green (1958) suggested that the two outputs should combined linearly:

$$
U=a U\left(f_{1}\right)+b U\left(f_{2}\right) .
$$

Since in signal detection under uncertainty there is usually no reason to weight the filters differently, it is reasonable to set $a=b=1$. But then it is easy to show that for a 2IFC procedure and an energy detector, the percentage of correct responses is normaly distributed with

$$
z=\frac{\left(E_{s} / N_{0}\right)}{\sqrt{2 M W T+2 E_{s} / N_{0}}},
$$

where $M$ reflects the number of filters. This result demonstrates that in the case considered here, doubling the width of a filter is mathematically equivalent to utilizing two independent and non-overlapping filters of equal width. Results obtained by widening a filter also apply interchangeably to this kind of multiplefilter model.

Another variant of the multiple-band model was suggested by Creelman (1960). In his two-filter model he assumed that maximum output of the filters determine the response. If Creelman's formula is generalized to more than two filters and adapted to a 2IFC method we find

$$
P\{C\}=\frac{M-1}{2 M-1}+\frac{M}{2 M-1} \int_{-\infty}^{\infty} f_{s}(x) F_{n}(x)^{2 M-1} d x .
$$

Increasing the number $M$ of filters again has the effect of increasing the slope of the psychometric functions. These results show that there are consistent fundamental differences between the two classes of models.

\section{Discussion}

Increasing filter bandwidth and changing filter-position at random have quite different effects on detectability. Although both processes result in loss of sensitivity, the slopes of the respective psychometric functions behave differently. Widening a filter steepens the slope, whereas randomly positioning the filter causes the slope to decrease. It should be possible to test these diverging predictions empiricaly.

One interesting application could be Green's (1961) suggestion that even with a single frequency there is frequency uncertainty. If this is indeed true then it is an 
empirical question whether such uncertainty can be accounted for by widening the auditory filter or by mistuning a filter with fixed bandwidth.

The results obained here also bear on the question of how cues work. Do they improve the filter tuning or do they cause the filter to narrow? By collecting psychometric functions while varying the effectiveness of the cues it should be possible to answer this question.

\section{ACKNOWLEDGMENTS}

This research was conducted while the author was a visiting scholar at the Psychology Department of the University of California at Berkeley, with support from the research council of NATO (administrated by the DAAD). The author is indebted to Professor Ervin R. Hafter for his supervision and his valuable suggestions. Thanks are also due to Dr. Robert Schlauch for his helpful comments. Finally, I thank Dr. Donald Bamber for his constructive criticisms and an unknown reviewer for improving the grammar of an earlier version of this paper.

\section{REFERENCES}

Creelman, C. D. (1960). Detection of signals of uncertain frequency. Journal of the Acoustical Society of America, 32, 805-810.

FelleR, W. (1966). An introduction to probability theory and its applications. Vol. II. New York: Wiley.

Fletcher, H. (1940). Auditory patterns. Review of Modern Physics, 12, 47-65.

Green, D. M. (1958). Detection of multiple component signals in noise. Journal of the Acoustical Society of America, 30, 904-911.

GreEN, D. M. (1960). Auditory detection of a noise signal. Journal of the Acoustical Society of America, 32, 121-131.

GrEEN, D. M. (1961). Detection of auditory sinosoids of uncertain frequency. Journal of the Acoustical Society of America, 33, 897-903.

Green, D. M., \& Swets, J. A. (1966). Signal detection theory and psychophysics. New York: Wiley.

GreEN, D. M., \& McGilL, W. J. (1970). On the equivalence of detection probabilities and well-known statistical quantities. Psychological Review, 77, 294-301.

Gundy, R. F. (1961). Auditory detection of an unspecified signal. Journal of the Acoustical Society of America, 33, 1008-1012.

von Helmholtz, H. L. F. (1863). Die Lehre von den Tonempfindungen als physiologische Grundlage für die Theorie der Musik. Braunschweig: Vieweg.

HogG, R. V., \& Craig, A. T. (1970). Introduction to mathematical statistics. London: Collie-Mcmillan.

JohNSON, D. M., \& HAFTER, E. R. (1980). Uncertain-frequency detection: Cueing and condition of observation. Perception \& Psychophysics, 28, 143-149.

MCGill, W. J. (1967a). Neural counting mechanisms and energy detection in audition. Journal of Mathematical Psychology, 4, 351-367.

McGill, W. J. (1967b). Variations on Marill's detection formula. Journal of the Acoustical Society of America, 43, 70-73.

Онм, G. S. (1943). Über die Definition des Tones, nebst daran gekniipfter Theorie der Sirene und ähnlicher tonbildender Vorrichtungen. Annalen für Physik und Chemie, 59, 513-565.

Patterson, R. D. (1974). Auditory filter shape. Journal of the Acoustical Sociely of America, 59, 802-809.

Patierson, R. D., \& Henning, G. B. (1977). Stimulus variability and auditory filter shape. Journal of the Acoustical Society of America, 62, 649-664. 
Patterson, R. D., \& Moore, B. C. J. (1986). Auditory filters and exitation patterns as representations of frequency resolution. In B. Moore (Ed.), Frequency selectivity in hearing (pp. 123-177). London: Academic Press.

Patterson, R. D., Nimmo-Smith, I., We8er, D. L., \& Milroy, R. (1982). The deterioration of hearing with age: Frequency selectivity, the critical ratio, the audiogram, and speech threshold. Journal of the Acoustical Society of America, 72, 1788-1803.

ShIfFrin, R. M. (1988). Attention. In R. C. Atkingon, R. J. Herrnstein, G. Lindzey, and R. D. Luce (Eds.), Stevens handbook of experimental psychology, Vol. 2 (pp. 739-811). New York: Wiley.

SHIPLEY, E. F. (1959). Cueing as a determinant of apparent variability in sensitivity. Journal of the Acoustical Society of America, 31, 834 (Abstract).

SwETs, J. A. (1963). Central factors in auditory frequency selectivity. Psychological Bulletin, 60, 429-440.

SwETs, J. A. (984). Mathematical models of attention. In R. Parasuraman and D. Davis (Eds.), Varieties of attention (pp. 183-242). New York/London: Academic Press.

Swets, J. A., \& Sewall, S. T. (1961). Stimulus versus response uncertainty in recognition. Journal of the Acoustical Society of America, 33, 1586-1592.

Swets, J. A., Green, D. M., \& TANner, W. P., JR. (1962). On the width of critical bands. Journal of the Acoustical Society of America, 34, 108-113.

Swets, J. A., Shipley, E. F., MCKey, M. J., \& Green, D. M. (1959). Multiple observations of signals in noise. Journal of the Acoustical Society of America, 31, 514-521.

Tanner, W. P., Jr., Swets, J. A., \& Green, D. M. (1956). Some general properties of the hearing mechanism. Ann Arbor, MI: University of Michigan.

VENIAR, F. A. (1958). Signal detection as a function of frequency ensemble. Journal of the Acoustical Sociely of America, 30, 548-557. 